\title{
Analysis on the Training Content and Method of Special Jump Skill of Volleyball Players in Middle School
}

\author{
Lei Wang ${ }^{1, a^{*}}$ and Wenmei Dong ${ }^{2, b}$ \\ ${ }^{1}$ Minzu University of ChinaNo.27 Zhongguancun South Main Street,Beijing, Haidian District, China \\ ${ }^{2}$ Minzu University of ChinaNo.27 Zhongguancun South Main Street,Beijing,Haidian District,China \\ awanglei666@muc.edu.cn, ${ }^{\mathrm{b}}$ dongwenm@163.com
}

Keywords: volleyball players; vertical jump quality; physiological anatomy; training

\begin{abstract}
This This article mainly uses the literature data method, the logical analysis meth od, the expert interview method and so on, combined with the author's research result, sum marizes and discusses the training content and the method research of the domestic high sc hool students volleyball athletes, Volleyball players special vertical jump ability training, sho uld be fast small power-based, do not use heavy weight exercise, training should be based on the athlete's physical condition, growth and development of the law to adjust the intensit $\mathrm{y}$ of training and time.
\end{abstract}

\section{Introduction}

In August 2016, the Chinese women's volleyball team beat the Serbian women's volleyball team, won the Rio Olympic women's volleyball champion. After the game Lang Ping said: "The spirit of the women's volleyball team is not to win the championship, but sometimes know will not win, but also make every effort. You all the way to go rickety, but stood up and shook the dust, still firmly in the eyes." The spirit of the women's volleyball team has deeply inspired the coaches and athletes who have been engaged in grass-roots volleyball training for a long time and have been making progress. Middle school volleyball players as the reserve force of our volleyball, the State Sports General Administration of the Center has always attached great importance to the movement in primary and secondary schools to carry out and promote each year the regional association will regularly organize various competitions and training, in order to promote grassroots Volleyball development, and then for our country to develop outstanding volleyball players.

With the rapid development of modern volleyball, volleyball competition in the online competition to become increasingly fierce, "high, fast" has become a high level of volleyball athletes basic quality. Athletes excellent jumping ability is to complete the volleyball storm, echelon attack, jump serve, jump pass and back attack and other technical and technical requirements of the basic skills. So the air high point often determine the spike, block the success rate, and even affect the outcome of the game. While the high point of the advantages in addition to the athletes congenital height factors, but also excellent jumping ability is to make up for lack of height, the key to winning the commanding heights. Therefore, it is very important to improve the vertical jump ability of the volleyball players. This paper mainly adopts the methods of literature, logic analysis, expert interview and so on. Based on the research results of the author, The method of research and so on to summarize and expound the purpose of understanding in detail the middle school students volleyball special vertical jump quality training methods and combined with foreign advanced training methods and means, and then for our middle school students volleyball players special vertical jump quality training content and methods to provide reference And explore new training patterns. 


\section{The Impact of Volleyball Athletes Vertical Jump Ability of Biomechanical Analysis}

Physiological Perspective on the Volleyball Player's Ability to Jump. In the middle school volleyball training, often using squat, squat and other traditional training methods to develop lower limb strength and jumping ability, coaches pay more attention to the increase in absolute muscle strength, the size of the absolute muscle strength and muscle cross-section, muscle cross-section The greater the absolute muscle strength, and the size of the muscle cross-section depends on the muscle muscle fiber number and the thickness of each muscle fiber, so the absolute muscle strength can only reflect the size of muscle strength and can not reflect the muscle muscle strength Of the size, so blindly asked the absolute strength, ignoring the volleyball player vertical jump ability on the lower limbs of various parts, joints and muscles in the starting speed, action amplitude, muscle rapid contraction, muscle explosive and other requirements. Although after practice, the practice of skeletal muscle physiology, anatomical cross-section increased, lower limb absolute muscle strength increased, but the rebound is not very significant increase.

Strength-velocity curves (in vitro muscles) show that, in other cases the same circumstances, the volleyball players want to make the muscles can quickly shrink, you must reduce the load, small load training can make muscle contraction speed is improved, if the muscle To overcome the larger load resistance, the contraction rate will slow down. Therefore, through different load training, you can make the body parts of the muscles to achieve the desired training effect.

An Analysis of the Influence of Anatomical Angle on Volleyball Player's Vertical Jumping Ability. When the athlete completes the volleyball technique in the spike and blockage and so on, the pelvis is moved from the forward tilt of the prepared take-off action, the hip joint, the knee joint is bent from the ready to take off, Flexion pedal extension, the lower limb muscle work is characterized by the first force to complete the anastomotic muscle contraction, and then the same group of rats quickly into isotropic to the contraction of the heart. This movement of the original muscle from the centrifugal contraction began to shrink to the heart of the work characteristics, can directly affect the human body when the jump speed and vacated height, while the volleyball player take off the height and swing arm speed also has a great relationship, Of the swing in the vertical jump action plays a synergistic and driving the role of lower limb force. Therefore, the full and rapid swing arm to play the lower limbs and waist strength, the maximum access to take off a certain degree of play.

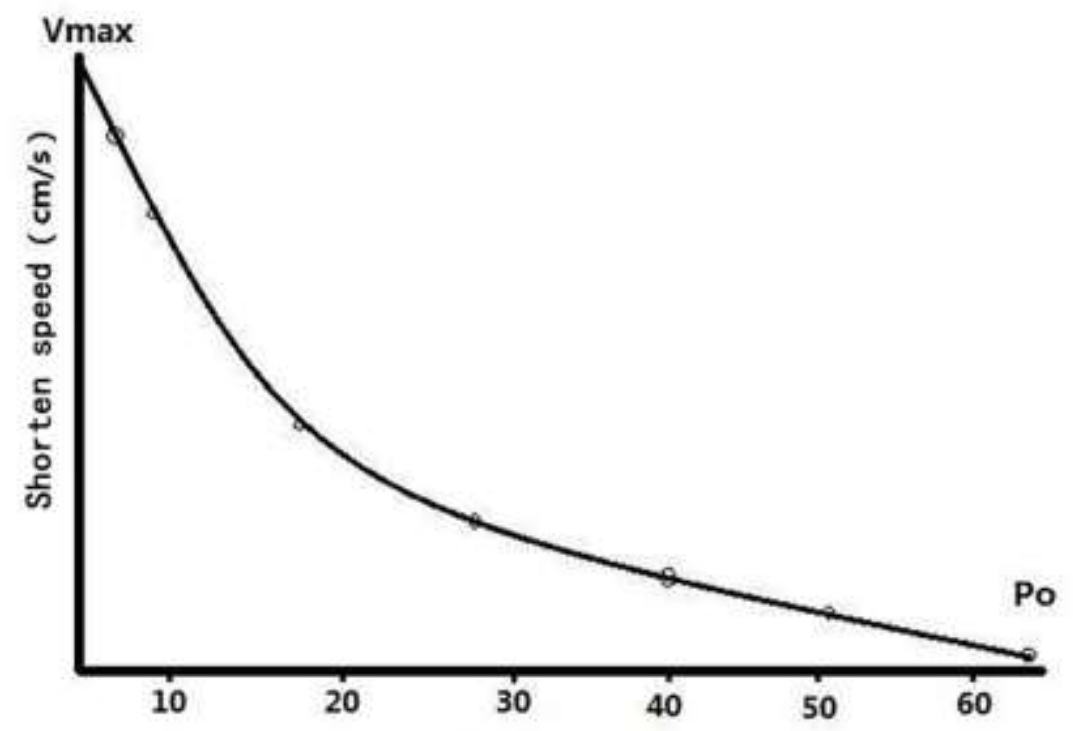

Fig. 1Force-speed diagram(Isolated muscle)

\section{Analysis of Physiological Characteristics of Middle School Students}

Adolescence is a critical period of human growth and development, and its growth and development is unbalanced, this period, the main features of its muscles are more water, protein, fat and inorganic salts less contraction function is weak, poor tolerance, fatigue, The main features of the bones are 
more cartilage tissue, bone tissue, water and organic ingredients more inorganic salts are less. So the hardness of the bones of small, toughness, not prone to fracture, but easy to bend deformation. At the same time, in this period of the athletes of the joints of the cartilage thicker, ligament stretch, so in this period, the joint range of activities than adults, but the firmness is poor, vulnerable. Duringthis period, the growth and development of muscle growth is not balanced, the body trunk muscle before the limb muscle, flexor before the extension of muscle, upper limb muscle first in the lower limb muscle, large muscle before the small muscle, in the growth phase, Longitudinal development, the strength of the fixed joint is weak. Bones are in the rapid growth stage, heavy load strength training, easy to cause joint damage, causing lower extremity deformation, but also may inhibit bone growth, resulting in the growth of young athletes affected. Therefore, if the increase in jumping force, it is necessary to increase the strength of the joints, in the development of flexibility at the same time, should strengthen the stability of the joint ligament, muscle strength exercises. In order to prevent muscle tissue hypertrophy or spine burden, bone deformity, bending, etc., in the training should develop the body of the muscles of the general strength of the various parts of the body, And more use of dynamic exercises, static exercises to be assisted, and more to overcome their own heavy practice or light equipment weight training and other small load training. Mainly through the internal coordination of bone and muscle tissue to increase the strength.

\section{Discussion on Training Contents and Methods}

According to the above analysis, the author thinks that the development of the students' volleyball players should avoid the heavy load training. The combination of "local" and "whole", "static" and "dynamic", "vertical jump quality and special ability" Mode to carry out training.

"Local and overall" exercise is: first on the lower limb strength, the core area of strength, upper arm swing arm speed, flexibility and other aspects of local training and then through a variety of training methods to integrate the above several parts of the practice, Static and dynamic "exercise is: in the training of the athletes in front of the original requirements of a combination of special features for a variety of vertical jump practice, and then in conjunction with the special conduct of a variety of longitudinal practice, and finally in the" dynamic"," static "combination, Practice.

In situ Slip Practice Method: Athletes on both sides of the body fixed elastic band, the end of the elastic band fixed to the ground, the athletes pedal to take off, the air to complete the abdomen practice or combined block action, take off after touching online volleyball.

Athletes in situ vertical jump, combined with the characteristics of volleyball, in place to complete the spike or the Nets technical action (no ball).

Athletes placed in front of a high platform (height should not be too high), athletes feet or feet in place to jump high platform.

Athletes side of the body placed a high platform, athletes standing on a high platform, one foot on the ground, the athlete to the other side of the swing pedal to take off, landing, the two leg exchange station, so repeated practice.

Athletes standing in front of the net, his hands touching the ground, even after jumping hands or one hand touch online fixed volleyball practice.

Combined with skipping practice, skipping when the athletes using single foot or feet, followed by continuous "single shake", "double shake", "three shake" practice.

Athletes in situ, around the continuous jump hurdle practice, the height of the column from low to high, the difficulty continues to increase until the maximum height.

Combined With Light Equipment, In Situ Vertical Jump Practice Method: Athletes holding hands on both hands, in situ vertical jump.

Athletes with both hands holding light equipment, in place for one foot or feet jump different height of the high platform to practice.

Athletes holding hands on the gravity ball over his head, and then hit the ball under the force, while the completion of longitudinal practice.

Travel Between The Vertical Practice Method: In the ground, in order to place $5-830 \mathrm{~cm}$ height of the column, the athletes continuous Jump the bar until the last jump after the completion 
of the fixed ball or fixed ball on the fixed ball practice.

Athletes step by step or feet jump step, jump steps can be increased in turn, so repeated practice.

Athletes standing on the high platform, and placed in front of the high column, the athletes jumped high platform, while swinging the pedal to jump the column, so repeat the practice.

Place the ladder in the venue, the athletes jump quickly for each ladder, the last jump to complete the spike or the Nets action.

Athletes in the sand, continuous before and after the completion of the left and right of the trip between the longitudinal practice. According to the frequent characteristics of volleyball vertical jump, spring training and volleyball needs to be combined with the special requirements of the volleyball, the development of back extensor, extensor muscle, flexion, flexor and toe muscle group explosive force.

\section{Conclusion}

The training of the students should be based on the physical condition of the athletes, the law of growth and development to adjust the training intensity load and time in time, and at the same time, In the daily training, the coaches should be more observation, more records, more analysis, more summary, through the "more than four" continuous innovation and optimization of training methods and means to enhance the athletes special jump ability.

\section{References}

[1] Journal of Shandong Sports Science, 2005, 27 (2): 95-96, 99 (in Chinese with English abstract).

[2] Wang Weimin, GuJiuxian.Effect of aerobic training and anaerobic training on the bouncing ability of volleyball players [J]. Journal of Nanjing Institute of Physical Education, 2004, 18 (6): 56.

[3] ZHANG Hai-tao Discussion on the training of volleyball players in spring [J]. Journal of Jianghan University, 2003 (1): 6-8.

[4] Shi Dasheng volleyball teaching and training guidance [M]. Beijing: People's Sports Publishing House, 1995: 102-105.

[5] Li Weiping, etc.Volleyball players in the strength of several training problems [J]. China Sports Science and Technology, 1999, (2): 40-42. 